\title{
Development of a real-time PCR for the detection of Chlamydia psittaci
}

Chlamydia psittaci is the causative agent of avian chlamydiosis, and zoonotic infection can result in severe pneumonia and other serious health problems (Stubbs et al., 1989). Even if this infection remains exceptional in humans, psittacosis can be considered as an emerging occupational disease (Lederer \& Muller, 1999; Maegawa et al., 2001). Direct detection of C. psittaci by cell culture is hazardous and requires a level 3 laboratory, given its contagiousness; currently, there is no other direct technique marketed. However, the interpretation of serodiagnosis is difficult because of cross-reactions with other species of Chlamydia and the high prevalence of Chlamydia pneumoniae in the general population. Therefore, molecular biology techniques represent invaluable tools for the diagnosis of this infection. The aim of this study was to develop a TaqMan PCR assay to detect $C$. psittaci by using the specific incA (inclusion membrane protein A) gene as a target. Since 1990, numerous standard PCR techniques have been developed for the detection and identification of C. psittaci. Most of them target major outer-membrane protein (MOMP) genes (Martin \& Cross, 1997; Sudler et al., 2004), as it is difficult to differentiate between species of Chlamydia based on the 16S rDNA. Recently several formats of real-time PCR have also been proposed for the identification and/or quantification of Chlamydia spp. They are usually very sensitive and rapid, and they avoid the possibility of contamination with PCR products since all of the steps occur in the same tube and no post-experimental handling is necessary. DeGraves et al. (2003) described a FRET (fluorescence resonance energy transfer)-PCR targeting the 23S rRNA gene that allows the identification of Chlamydia species. Two real-time PCRs targeting the ompl gene and using FRET or SYBR Green chemistries have been developed (Huang et al., 2001). No real-time PCR using TaqMan chemistry has been described to date. In this study, we used TaqMan methodology to develop a PCR for the detection of C. psittaci, in order to employ only one probe instead of two in FRET chemistry, and to shorten the size of amplicon, thus optimizing PCR efficiency. IncA is a protein synthesized and secreted onto the inclusion membrane by bacteria (Bannantine et al., 1998). It was first identified in 'Chlamydia caviae' strain GPIC, and homologues of the gene were found in all the chlamydial genomes sequenced. All IncA proteins share the same organization, but in spite of the structural conservation, there is little similarity in the sequences of IncA between different species (Delevoye et al., 2004). This observation prompted us to design C. psittaci-specific primers and probe based on the incA gene.

Several Chlamydia species such as $C$. pneumoniae TWAR strain AR39 (ATCC 53592), Chlamydia trachomatis strain D/UW-3/Cx (ATCC VR-885), 'C. caviae' strain GPIC, 'Chlamydia abortus' strains AV2 and AV7, Chlamydia pecorum strains IB2 and IB5, and C. psittaci strains GR9, VS1, 225, NJ1 and CP-3, kindly supplied by A. Rodolakis (INRA, Roumilly, France), were used in this study. They were cultured on appropriate cells in minimal essential medium supplemented with $1 \mathrm{mM}$ glutamine and $5 \%$ fetal bovine serum. Genomic DNA was extracted using a MagNA Pure LC DNA isolation kit I and a MagNA Pure LC isolation station (Roche Applied Science). DNA was stored at $-20^{\circ} \mathrm{C}$.

First, we sequenced the incA gene from different $C$. psittaci strains. Because the chlamydial strain sequenced that is closest to C. psittaci is ' $C$. abortus', PCR primers were designed in the genes flanking the 'C. abortus' incA gene (CAB536, according to 'C. abortus' annotation, GenBank accession number CR848038): CA-CAB535-S (5'-AAGGTCTCCTCCCATAATTGAG-3') and CA-CAB537-AS (5'-GAACGGTGCTTTTTATTGGAAA- $3^{\prime}$ ). The products of PCR amplification of genomic DNA from five different strains of $C$. psittaci were purified using MicroSpin S-400 HR columns (Amersham Pharmacia). Sequencing of these products was achieved on both strands using the initial CA-CAB535-S and CA-CAB537-AS primers, and internal primers designed within the sequences (IncA-Int2 5' -CGAAACCTTAACCTCAGGAA-3' and IncA-IntAS 5'-GAGTACCTACAGATTTGAAA-3'). The incA sequences of GR9, VS1, 225, NJ1 and CP3 strains were submitted to GenBank and assigned accession numbers DQ117471-DQ117475, respectively. Alignment of incA genes from 'C. abortus', 'C. caviae' and C. trachomatis with the five newly sequenced incA genes of $C$. psittaci showed that C. psittaci and 'C. abortus' incA genes were the most closely related, and that C. trachomatis and ' $C$. caviae' were more distant from each other (data not shown); this is in agreement with phylogenic trees based on 16S rRNA and 23S rRNA genes (Everett et al., 1999).

Second, we designed TaqMan primers and a probe for PCR. The five C. psittaci incA genes and the 'C. abortus' incA gene were aligned using the multiple sequence alignment with hierarchical clustering program (Corpet, 1988), http:// prodes.toulouse.inra.fr/multalin/ multalin.html, and a search was carried out to identify conserved regions specific for C. psittaci. Primers and probes were designed using the Primer Express software package (Applied Biosystems) and were synthesized by Eurogentec. The primers (F1-incA-Cpsi 5'-GCCATCATGCTTGTTTCGTTT-3' and R1-incA-Cpsi 5'-CGGCGTGCCACTTGAGA-3') were designed to amplify a $74 \mathrm{bp}$ region on the C. psittaci incA gene (Fig. 1). The probe was designed to match a sequence conserved in the five $C$. psittaci sequences but with one mismatch with the ' $C$. abortus' sequence to ensure specificity (Cpsi-incA-NM, FAMTCATTGTCATTATGGTGATTCAGGANFQ-MGB). The MGB (minor groove binder) TaqMan probe was synthesized 
C. psittaci GR9

C. psittaci 225

C. psittaci VS1

C. psittaci NJ1

C. psittaci CP3

C. abortus $526 / 3$

Consensus
TGATTGCCATCATGCTTGTTTCGTTTGTCATTGTCATTATGGTGATTCAGGATAGCACCCCCTCTCAAGTGGCACGCCGTATGA TGATTGCCATCATGCTTGTTTCGTTTGTCATTGTCATTATGGTGATTCAGGATAGCACCCCCTCTCAAGTGGCACGCCGTATGA TGATTGCCATCATGCTTGTTTCGTTTGTCATTGTCATTATGGTGATTCAGGATAGCACCCCCTCTCAAGTGGCACGCCGTATGA TGATTGCCATCATGCTTGTTTCGTTTGTCATTGTCATTATGGTGATTCAGGATAGCACCCCCTCTCAAGTCGCACGCCGTATGA TGATTGCCATCATGCTTGTTTCGTTTGTCATTGTCATMATGGTGATTCAGGATAGCACTCCCTCTCAAGTGGCACGCCGTATGA

TGATTGCCATTATGCTCGTTTCGTTTGTCATTGTCATMATGGTCATTCAGGATAGCACACCTTCTCAAGTGGCGCGTCGAATGA TGATTGCCATCATGCTLGTTTCGTTTGTCATTGTCATTATGGTgATTCAgGATAGCAC. CCCTCTCAAGTgGCaCGCCGtATGA

Fig. 1. Representation of the nucleotide sequence from the inc $A$ gene of five $C$. psittaci strains and one ' $C$. abortus' strain, $\mathrm{S} 26 / 3$, and location of the primers and probe used for real-time PCR amplification. The sequences are shown from nucleotide 305 up to nucleotide 388 . Primers are underlined and the probe is boxed. Mismatches are indicated in bold.

with the reporter dye FAM (6-carboxyfluorescein) covalently linked to the $5^{\prime}$ ends, and the non-fluorescent quencher, NFQ, at the $3^{\prime}$ ends, which were phosphorylated to prevent probe extension. The primers and probe were tested on DNA extracted from the five C. psittaci strains, five other Chlamydia species, and from diverse bacterial species commonly found in human specimens, including Gram-negative bacteria (Escherichia coli, Salmonella enteritidis, Salmonella typhimurium, Neisseria meningitidis, Haemophilus influenzae, Haemophilus parainfluenzae, Bordetella pertussis, Bordetella parapertussis, Bordetella holmesii) and Gram-positive and related bacteria (Mycoplasma pneumoniae, Mycoplasma genitalium, Streptococcus pneumoniae, Staphylococcus aureus). The TaqMan real-time PCR amplification and hybridization reactions were carried out in a final volume of $25 \mu \mathrm{l}$ containing $12 \cdot 5 \mu \mathrm{l}$ TaqMan Universal PCR master mix, $0 \cdot 3 \mu \mathrm{M}$ each primer, $0 \cdot 2 \mu \mathrm{M}$ labelled probe Cpsi-incA-FT and $5 \mu \mathrm{l}$ purified DNA in a ABI PRISM 7000 thermocycler (Applied Biosystems). DNA was amplified using the following cycling parameters: heating at $95^{\circ} \mathrm{C}$ for $10 \mathrm{~min}$, followed by 40 cycles of a two-stage temperature profile of $95^{\circ} \mathrm{C}$ for 15 s and $60^{\circ} \mathrm{C}$ for $1 \mathrm{~min}$. Only DNA extracted from C. psittaci led to amplification, demonstrating the specificity of the method.

To evaluate sensitivity, infectious bacteria, called elementary bodies, were purified (Scieux et al., 1993), and quantification was performed by diluting the bacterial suspension in PBS and counting the elementary bodies after staining with the direct fluorescent monoclonal antichlamydia antibody (IMAGEN Chlamydia antibody; J2L Elitech). Serial dilutions of the extracted DNA from bacterial suspensions were tested using this assay (Fig. 2). The slope of the regression curve was -3.524 i.e. very close to $-3 \cdot 32$ corresponding to the maximum efficiency. The regression curve was linear from $10^{6}$ to 1 bacterial genome for a reaction volume of $25 \mu \mathrm{l}$.

This PCR was applied to two clinical samples (broncho-alveolar lavages) from patients presenting severe respiratory infections and being positive by serology. One patient worked in a poultry abattoir and the other patient was a parakeet breeder. These two samples were positive by TaqMan PCR. In conclusion, we have (a)

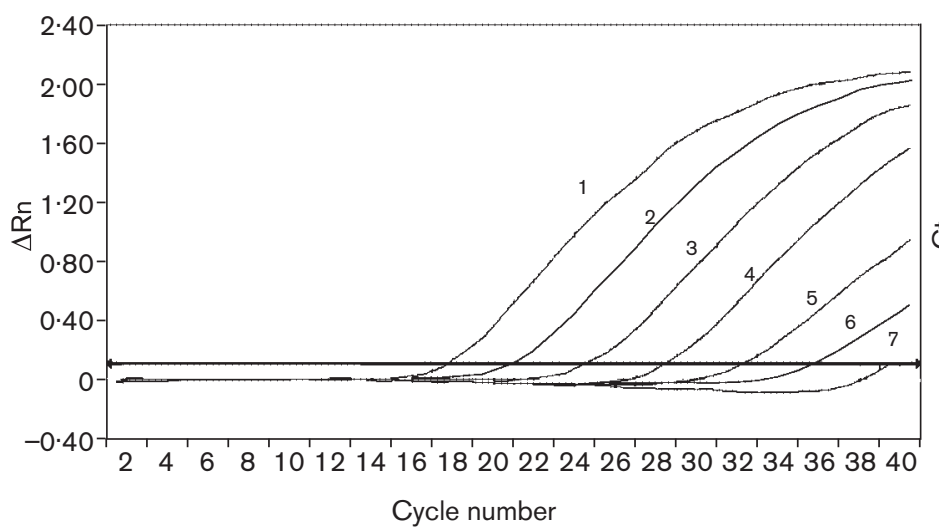

(b)

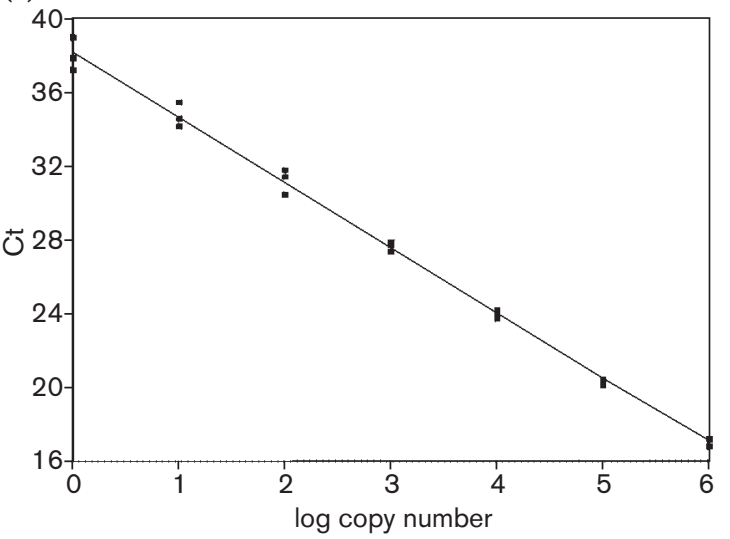

Fig. 2. Amplification plots and deduced standard curve. (a) Example of amplification plots from 1 to 7 generated from $10^{6}$, $10^{5}, 10^{4}, 10^{3}, 10^{2}, 10$ and 1 bacteria, respectively. The PCR cycle numbers are indicated on the $x$ axis, and the corresponding $\Delta \mathrm{Rn}$ (magnitude of the signal generated by the specified set of PCR conditions) are given on the $y$ axis. Fluorescence $=f$ (cycles). (b) A standard curve was generated from each sample tested in triplicate. The copy numbers of $C$. psittaci genomic DNA used for the standard curve are indicated on the $x$ axis, and the corresponding Ct (fractional cycle number at which the fluorescence passes the threshold) values are given on the $y$ axis. $\mathrm{Ct}=\mathrm{f}(\log \mathrm{copy}$ number). $y=-3.524 \times \log (x)+38 \cdot 12$. The $r 2$ value from the linear regression in this assay is 0.99 . 
developed a new real-time PCR for the identification of $C$. psittaci that we believe is the first to be applied successfully to human samples. This method is rapid, sensitive and specific, and can be easily implemented in a setting where a real-time TaqMan-PCR apparatus is available.

\section{Acknowledgements}

The authors want to thank A. Rodolakis from the Pathologie Infectieuse et Immunologie Laboratoire, INRA, Roumilly, France, for supplying 'C. caviae', 'C. abortus', C. pecorum and C. psittaci strains.

\section{Armelle Ménard, ${ }^{1}$ Maithé Clerc, ${ }^{2}$ Agathe Subtil, ${ }^{3}$ Francis Mégraud, ${ }^{1}$ Christiane Bébéar ${ }^{2}$ and Bertille de Barbeyrac ${ }^{2}$ \\ 1,2INSERM ERI 10 Laboratoire de Bactériologie $^{1}$ and Centre National de Référence des Chlamydia, Laboratoire de Bactériologie ${ }^{2}$, Université Victor Segalen Bordeaux 2, Bordeaux, France \\ ${ }^{3}$ Unité de Biologie des Interactions Cellulaires, Institut Pasteur, CNRS URA 2582, Paris, France}

Correspondence: Bertille de Barbeyrac (Bertille.de.Barbeyrac@u-bordeaux2.fr)
Bannantine, J. P., Stamm, W. E., Suchland, R. J. \& Rockey, D. D. (1998). Chlamydia trachomatis IncA is localized to the inclusion membrane and is recognized by antisera from infected humans and primates. Infect Immun 66, 6017-6021.

Corpet, F. (1988). Multiple sequence alignment with hierarchical clustering. Nucleic Acids Res 16, 10881-10890.

DeGraves, F. J., Gao, D., Hehnen, H. R., Schlapp, T. \& Kaltenboeck, B. (2003). Quantitative detection of Chlamydia psittaci and C. pecorum by high-sensitivity real-time PCR reveals high prevalence of vaginal infection in cattle. J Clin Microbiol 41, 1726-1729.

Delevoye, C., Nilges, M., Dautry-Varsat, A. \& Subtil, A. (2004). Conservation of the biochemical properties of IncA from Chlamydia trachomatis and Chlamydia caviae: oligomerization of IncA mediates interaction between facing membranes. J Biol Chem 279, 46896-46906.

Everett, K. D., Bush, R. M. \& Andersen, A. A. (1999). Emended description of the order Chlamydiales, proposal of Parachlamydiaceae fam. nov. and Simkaniaceae fam. nov., each containing one monotypic genus, revised taxonomy of the family Chlamydiaceae, including a new genus and five new species, and standards for the identification of organisms. Int J Syst Bacteriol 49, $415-440$.
Huang, J., DeGraves, F. J., Gao, D., Feng, P., Schlapp, T. \& Kaltenboeck, B. (2001).

Quantitative detection of Chlamydia spp. by fluorescent PCR in the LightCycler. Biotechniques 30, 150-157.

Lederer, P. \& Muller, R. (1999). Ornithosis studies in correlation with an outbreak. Gesundheitswesen 61, 614-619 (in German).

Maegawa, N., Emoto, T., Mori, H., Yamaguchi, D., Fujinaga, T., Tezuka, N., Sakai, N., Ohtsuka, N. \& Fukuse, T. (2001). Two cases of Chlamydia psittaci infection occurring in employees of the same pet shop. Nihon Kokyuki Gakkai Zasshi 39, 753-757 (in Japanese).

Martin, J. L. \& Cross, G. F. (1997). Comparison of the omp I gene of Chlamydia psittaci between isolates in Victorian koalas and other animal species. Aust Vet J 75, 579-582.

Scieux, C., Grimont, F., Regnault, B., Bianchi, A., Kowalski, S. \& Grimont, P. A. (1993). Molecular typing of Chlamydia trachomatis by random amplification of polymorphic DNA. Res Microbiol 144, 395-404.

Stubbs, R., Dralle, W. \& Williams, J. (1989). Psittacosis pneumonia. J Tenn Med Assoc 82, 189-190.

Sudler, C., Hoelzle, L. E., Schiller, I. \& Hoop, R. K. (2004). Molecular characterisation of chlamydial isolates from birds. Vet Microbiol 98, 235-241. 